TRANSACTIONS OF THE

AMERICAN MATHEMATICAL SOCIETY

Volume 349, Number 10, October 1997, Pages 3911-3923

S 0002-9947(97)01530-4

\title{
DEGENERATE PARABOLIC EQUATIONS WITH INITIAL DATA MEASURES
}

\author{
DANIELE ANDREUCCI
}

\begin{abstract}
We address the problem of existence of solutions to degenerate (and nondegenerate) parabolic equations under optimal assumptions on the initial data, which are assumed to be measures. The requirements imposed on the initial data are connected both with the degeneracy of the principal part of the equation, and with the form of the nonlinear forcing term. The latter depends on the space gradient of a power of the solution. Applications to related problems are also outlined.
\end{abstract}

\section{INTRODUCTION AND MAIN RESUlTS}

We consider existence (and nonexistence) of solutions to the initial value problem for degenerate parabolic equations, when the initial datum is a prescribed Radon measure $\mu$ in $\mathbf{R}^{N}, N \geq 1$. The $L^{\infty}$ estimates we prove for such solutions seem to be new even in the case of the homogeneous porous media equation, but our methods apply to a wide class of degenerate parabolic equations with nonlinear sources, and even to weakly coupled systems of such equations. However, we have chosen as a model problem

$$
\begin{aligned}
u_{t}-\Delta u^{m} & =\left|D u^{p}\right|^{\nu}, \quad \text { in } S_{T} \stackrel{\text { def }}{=} \mathbf{R}^{N} \times(0, T), T>0, \\
u(x, 0) & =\mu, \quad x \in \mathbf{R}^{N},
\end{aligned}
$$

where $D v=\left(v_{x_{1}}, \ldots, v_{x_{N}}\right)$, and we assume throughout this paper $m \geq 1, p \nu \geq m$, $0<\nu<2, N \geq 1$ (any $\nu \geq 1$ is allowed in Theorem 1.2). In (1.1), $u$ is assumed to be nonnegative, and $\mu$ denotes a Radon measure. Generalisations are collected in Remarks 1.1, 1.2 below. Here we only note that our methods allow one to prove existence of solutions, corresponding to data measures, for

$$
u_{t}-\Delta u^{m}=u^{a}, \quad \text { in } S_{T},
$$

even in the supercritical case $a>m+2 / N$.

Let us briefly explain our motivations. It is well known (see, e.g., [7]) that the Cauchy problem for

$$
u_{t}-\Delta u^{m}=0, \quad \text { in } S_{T},
$$

is solvable for any (e.g., finite) Radon measure $\mu$ in (1.1b). As far as we know, the problem of existence of solutions to (1.2)-(1.1b), $\mu$ a measure, was still open, at

Received by the editors August 15, 1994 and, in revised form, March 20, 1995.

1991 Mathematics Subject Classification. Primary 35K65, 35R05; Secondary 35K55, 35K15.

The author is a member of GNFM of Italian CNR. Work supported by MURST project "Problemi non lineari..."

(C)1997 American Mathematical Society 
least in the case $a>m+2 / N, m>1$. This kind of problem is closely connected with the derivation of a priori sup estimates of the solution sharply depending on the regularity of the initial datum (to be understood in a suitable sense).

In order to clarify this point, let us state the following straightforward consequence of the well known Harnack inequality for (1.3) (see [5]). Let $u$ be a continuous nonnegative supersolution to (1.3), and let $u(\cdot, t) \rightarrow \mu$ as $t \rightarrow 0$, in the sense of measures, with

$$
\mu\left(B_{\rho}(0)\right) \geq \varepsilon \rho^{N-\vartheta}, \quad 0<\rho<\sigma,
$$

where $\sigma>0, \varepsilon>0,0 \leq \vartheta \leq N$ are given, and we set $B_{\rho}(z)=\{|x-z|<\rho\}$. Then (see Lemma 2.1 in [2])

$$
u(x, t) \geq \gamma_{0} \varepsilon^{\frac{2}{2+\vartheta(m-1)}} t^{-\frac{\vartheta}{2+\vartheta(m-1)}},
$$

for $|x| \leq \gamma_{0} \varepsilon^{\frac{m-1}{2+\vartheta(m-1)}} t^{\frac{1}{2+\vartheta(m-1)}}<\sigma$ (in (1.4b) $\gamma_{0}$ depends on $N, m, \vartheta$; below we denote by $\gamma, \gamma_{0}$ generic positive constants).

Roughly speaking, we show in the sequel that (1.4a) still implies (1.4b), if both inequalities are reversed; this is true (under some assumptions on $\vartheta$ ) for solutions to (1.1a), to (1.2), or to (1.3). (In the case $\vartheta=N$, this was known, for (1.3) [7], and for (1.2) [4], when $a<m+2 / N$.) Our approach relies upon suitable a priori estimates, in the spirit of [2], [3], [4]. But, for "large" exponents in the source terms, the just mentioned papers obtain well behaved estimates at the expense of higher integrability of the solution (and therefore of the initial datum). In contrast, here we are able to circumvent this difficulty, essentially by choosing the correct geometry for the domains involved in our estimates. Then, the latter involve just (a local version of) the $L^{1}$ norm of the solution, as well as of the initial datum.

Concerning (1.1a), we quote the paper [12], dealing with semilinear parabolic equations of higher order, whose results cover equation (1.1a) in the special case $m=p=1, \nu>1$. We also recall the extensive work of Amann (see e.g., [1]) about general nonlinear nondegenerate parabolic equations and systems. Both approaches seem to require that the initial datum belongs to a suitable space $L^{q}$, $q>1$. Semilinear problems somehow related to (1.1) are also treated in [17].

We stress the fact that, as far as we know, we give here for the first time a proof of the optimality of the regularity requirements we stipulate on the initial datum. Our interest is mainly focussed on the local regularity of $\mu$ in (1.1b). See [11] for an investigation of the admissible behaviour at infinity of smooth initial data to a one-dimensional equation related to (1.1a).

Finally, let us note that, to the best of our knowledge, the question of uniqueness of solutions to (1.1) is still open.

Definition 1.1. A solution to (1.1a)-(1.1b) is $u \in L_{\mathrm{loc}}^{\infty}\left(S_{T}\right) \cap C\left((0, T) ; L_{\mathrm{loc}}^{2}\left(\mathbf{R}^{N}\right)\right)$, $u \geq 0$, a function with $\left|D u^{m}\right|^{2},\left|D u^{p}\right|^{\nu} \in L_{\text {loc }}^{1}\left(S_{T}\right)$, such that

$$
\iint_{S_{T}}\left\{-u \varphi_{t}+D u^{m} \cdot D \varphi\right\} d x d t=\iint_{S_{T}}\left|D u^{p}\right|^{\nu} \varphi d x d t
$$

for all $\varphi \in C_{0}^{1}\left(S_{T}\right)$. Moreover we require that $u(\cdot, t) \rightarrow \mu$ as $t \rightarrow 0$ in the sense of measures. Subsolutions and supersolutions to (1.1a) are defined by standard modifications of (1.5).

It follows from the results given in [9], and from the techniques employed therein, that solutions in the sense above are actually Hölder continuous in $S_{T}$. 
Notation. Let $\mu$ be any Radon measure in $\mathbf{R}^{N}$, and $u \in L_{\text {loc }}^{\infty}\left(S_{T}\right), u \geq 0$, with $S_{T}=\mathbf{R}^{N} \times(0, T), T>0$. Let also $N \geq \vartheta \geq 0$ be given. We use the following notation throughout the paper:

$$
[\mu]=\sup _{x \in \mathbf{R}^{N}} \sup _{0<\rho<1} \rho^{\vartheta} f_{B_{\rho}(x)} d \mu, \quad[u]_{t}=\sup _{0<\tau<t}[u(\cdot, \tau)], \quad 0<t<T,
$$

where we let

$$
f_{E} d \mu=\frac{1}{|E|} \int_{E} d \mu, \quad|E|=\text { Lebesgue-meas }(E) .
$$

First we state our existence result.

Theorem 1.1. Let $[\mu]$ be finite, with $\vartheta(p \nu-m)<(2-\nu)$. Then there exists a solution to (1.1) defined in $\mathbf{R}^{N} \times\left(0, T_{0}\right)$, where $T_{0}=T_{0}([\mu], N, m, p, \nu, \vartheta)$, such that for $0<t<T_{0}$ we have both $[u]_{t} \leq \gamma[\mu]$, and

$$
\|u(\cdot, t)\|_{\infty, \mathbf{R}^{N}} \leq \gamma t^{-\frac{\vartheta}{2+\vartheta(m-1)}}[\mu] .
$$

Here $\gamma=\gamma(N, m, p, \nu, \vartheta)$.

Next we prove that the critical threshold for $\vartheta$ made explicit in Theorem 1.1 is actually optimal for the existence of solutions in the class considered here. In the following we denote $s_{+}=\max (s, 0)$.

Theorem 1.2. Let $\nu \in[1, \infty)$. Let $u$ be a solution to $(1.1)$ in $\mathbf{R}^{N} \times(0, T)$ such that $[u]_{t}<\infty, 0<t<T$, and

$$
\mu\left(B_{\rho}\left(x_{0}\right)\right) \geq \varepsilon \rho^{N-\vartheta}, \quad 0<\rho<\varepsilon,
$$

where $\varepsilon>0, x_{0} \in \mathbf{R}^{N}$ are given. Then $\vartheta(p \nu-m) \leq(2-\nu)_{+}$.

We remark that assumption $[\mu]_{t}<\infty$ is redundant if $\vartheta=N$ : indeed this estimate is the content of the Harnack inequality quoted above. We also remark here that the positivity of the right-hand side of (1.1a) is exploited in the proof of Theorem 1.2. Of course, by the same token, solutions corresponding to nonnegative initial data can be found as limits of smooth positive solutions to approximating problems, as in Section 3 below. Let us also point out that the problem of existence of solutions in the limiting case $\vartheta(p \nu-m)=(2-\nu)$ is left open.

Remark 1.1 (Generalisations). All the priori estimates collected in Section 2 are valid for nonnegative solutions to

$$
u_{t}-\operatorname{div} \mathbf{a}\left(x, t, u, D u^{m}\right)=\left|D u^{p}\right|^{\nu},
$$

under standard structure assumptions on a. Minor changes in the proofs show that similar estimates can be proven for solutions to (1.3), (1.2) (or to equations of more general structure, as in (1.6)). Therefore, one can combine the techniques demonstrated below with the arguments in [4] (case of a space-dependent source), [2] (weakly coupled systems of equations), to get new existence results for equationsor for systems of equations - with sources of power type (see also the following remark).

Finally we note that our methods also yield existence of solutions of variable sign, approaching a signed measure $\mu$ as $t \rightarrow 0$, because the estimates below hold separately for the positive and negative parts of solutions. Of course in this case we understand the equations above by the convention $u^{s}=|u|^{s-1} u, s>0, u \in \mathbf{R}$, and we replace $\mu$ with its total variation $|\mu|$ in the definition of $[\mu]$. 
Remark 1.2 (About equation (1.2)). It is known ([4]) that any initial datum $\mu$ to a nonnegative solution of (1.2) must fulfill the following necessary condition:

$$
\mu\left(B_{\rho}(z)\right) \leq \gamma \rho^{N-\Lambda}, \quad \rho>0 \text { small, for all } z \in \mathbf{R}^{N},
$$

where $\Lambda=2 /(a-m)$. Of course (1.7) does not place any restriction on the local regularity of $\mu$ if $a<m+2 / N$, in which case to any (e.g., finite) Radon measure corresponds a solution to (1.2). As a new result of the techniques introduced here, we can prove that, even in the case $a>m+2 / N,(1.2)$ has a solution taking on an initial datum $\mu$ measure, provided $[\mu]<\infty$, with $\vartheta$ such that $a<m+2 / \vartheta$ (this bound is optimal by (1.7)).

In the case when the principal part of equation (1.2) is replaced by the standard heat operator, we quote the papers [6], [13], [14], [16]. We refer to [10], [14], [17] and to the literature quoted therein, for a discussion of the Morrey norms $[\mu]$ defined above, whose connection with initial value problems (mainly for semilinear parabolic equations) has been recently the subject of considerable interest. Parabolic equations with measure data are also treated in [8].

The plan of the paper is as follows: in Section 2 we collect the a priori estimates needed to prove Theorem 1.1, whose proof is given in Section 3. Theorem 1.2 is proven in Section 4.

\section{The A PRIORI ESTIMATES}

For technical reasons we need define, for $u \in L_{\mathrm{loc}}^{\infty}(S), u \geq 0$, with $S=\mathbf{R}^{N} \times$ $\left(0, T^{*}\right)$

$$
\langle u\rangle_{t}=\sup _{0<\tau<t} \sup _{x \in \mathbf{R}^{N}} \sup _{R(\tau)<\rho<1} \rho^{\vartheta} f_{B_{\rho}(x)} u(y, t) d y, \quad R(t)=\Gamma t^{\frac{1}{2+\vartheta(m-1)}},
$$

for all $0<t<T^{*}$, where $\Gamma$ is a positive constant which can be chosen a priori (as a function of $N, m, p, \vartheta, \nu$ and of $[\mu]$ ), and which will be specified later. Of course we need also assume that, e.g.,

$$
T^{*} \text { is chosen so that } R\left(T^{*}\right)=1 \text {. }
$$

Note that the last assumption is meaningful because $\Gamma$ is independent of $T^{*}$. The connection between $[u]_{t}$ and $\langle u\rangle_{t}$ is commented upon in Remark 2.2 below. Finally we define the constants

$$
\mathcal{K}=N(m-1)+2, \quad \Theta=\frac{\nu}{2-\nu}(2 p-m)-1 .
$$

We can check at once that assumption $\vartheta(p \nu-m)<(2-\nu)$ may be written as $\vartheta \Theta<(2+\vartheta(m-1))$, and that $\Theta>0$ follows from our assumptions.

The following sup estimate will be instrumental in proving our existence result.

Lemma 2.1. Let $u$ be a continuous nonnegative subsolution of (1.1a) in S. Assume also that a time $0<T<T^{*}$ is given so that

$$
\Gamma^{-2} t^{\frac{\vartheta}{2+\vartheta(m-1)}}(m-1)\|u(\cdot, t)\|_{\infty, \mathbf{R}^{N}}^{m-1}+t\|u(\cdot, t)\|_{\infty, \mathbf{R}^{N}}^{\Theta} \leq 1, \quad 0<t<T .
$$

Then

$$
\|u(\cdot, t)\|_{\infty, \mathbf{R}^{N}} \leq \gamma t^{-\frac{\vartheta}{2+\vartheta(m-1)}} \Gamma^{\frac{2}{\mathcal{K}}(N-\vartheta)}\langle u\rangle_{t}^{\frac{2}{K}}, \quad 0<t<T .
$$

Here $\gamma=\gamma(N, m, p, \nu)$. 
Proof. 1) Fix a ball $B_{\rho} \subset \mathbf{R}^{N}$, and $0<t<T$, with $R(t) \leq \rho$. Let $\left\{k_{n}\right\}$ be defined by $k_{n}=k\left(1-2^{-n-1}\right)$, for all $n \geq 0$, with $k>0$ to be chosen. Let also $\left\{Q_{n}\right\}$ be a suitable sequence of cylinders approaching (from the outside) $Q_{\infty}=B_{\rho / 2} \times(t / 2, t)$. Choose as a testing function in (1.5) $\eta=\left(u-k_{n}\right)_{+} \zeta_{n}^{2}, \zeta_{n}$ being a standard cutoff function in $Q_{n}$, such that $\zeta_{n} \equiv 1$ in $Q_{n+1}$. Then we estimate the right-hand side of (1.5) by Young's inequality, getting

$$
\begin{aligned}
\iint_{Q_{n}} & \left|D u^{p}\right|^{\nu}\left(u-k_{n}\right)_{+} \zeta_{n}^{2} d x d \tau \\
\leq & \varepsilon \iint_{Q_{n}}\left|D\left(u-k_{n}\right)_{+}\right|^{2} u^{m-1} \zeta_{n}^{2} d x d \tau \\
& +\gamma(\varepsilon) \iint_{Q_{n}} u^{\frac{\nu}{2-\nu}(2 p-m-1)}\left(u-k_{n}\right)_{+}^{1+\frac{\nu}{2-\nu}} \zeta_{n}^{2} d x d \tau \\
\leq & \varepsilon \iint_{Q_{n}}\left|D\left(u-k_{n}\right)_{+}\right|^{2} u^{m-1} \zeta_{n}^{2} d x d \tau \\
& +\gamma(\varepsilon) 2^{2 n}\|u\|_{\infty, Q_{n}}^{\Theta} \iint_{Q_{n}}\left(u-k_{n-1}\right)_{+}^{2} d x d \tau .
\end{aligned}
$$

Standard calculations then prove that the integral multiplied by $\varepsilon$ in (2.3) may be absorbed in the left-hand side of (1.5), and that

$$
\begin{aligned}
& \sup _{0<\tau<t} \int_{B_{\rho}}\left[\left(u-k_{n}\right)_{+} \zeta_{n}\right]^{2}(x, \tau) d x+k^{m-1} \iint_{Q_{n}}\left|D\left(u-k_{n}\right)_{+} \zeta_{n}\right|^{2} d x d \tau \\
& \leq 2^{2 n} \frac{\gamma}{t}\left\{1+\frac{t}{\rho^{2}}\|u\|_{\infty, Q_{n}}^{m-1}+t\|u\|_{\infty, Q_{n}}^{\Theta}\right\} \iint_{Q_{n}}\left(u-k_{n-1}\right)_{+}^{2} d x d \tau \\
& \leq 2^{2 n} \frac{\gamma}{t} \iint_{Q_{n}}\left(u-k_{n-1}\right)_{+}^{2} d x d \tau \quad \text { (by (2.1). }
\end{aligned}
$$

The recursive inequality (2.4) allows us to develop an iteration process eventually leading to

$$
\|u(\cdot, t)\|_{\infty, B_{\rho / 2}} \leq \gamma t^{-\frac{N}{\mathcal{K}}}\left(f_{0}^{t} \int_{B_{\rho}} u d x d \tau\right)^{\frac{2}{\mathcal{K}}}, \quad 0<t<T
$$

$\mathcal{K}=N(m-1)+2$. Here $\gamma=\gamma(N, m, p, \nu)$. We refer the reader to [3], without reproducing the details here.

2) For all $0<t<T, B_{\rho} \subset \mathbf{R}^{N}, \rho=R(t)$, we get by virtue of (2.5)

$$
\|u(\cdot, t)\|_{\infty, B_{\rho / 2}} \leq \gamma t^{-\frac{N}{\mathcal{K}}}\left(f_{0}^{t} \int_{B_{R(t)}} u d x d \tau\right)^{\frac{2}{\mathcal{K}}} \leq \gamma t^{-\frac{N}{\mathcal{K}}} R(t)^{\frac{2}{\mathcal{K}}(N-\vartheta)}\langle u\rangle_{t}^{\frac{2}{\mathcal{K}}}
$$

whence (2.2) by plugging in the definition of $R(t)$.

The second ingredient we need is an estimate of the term containing $\left|D u^{p}\right|^{\nu}$ in

Lemma 2.2. Let the assumptions of Lemma 2.1 be fulfilled. For $\lambda \in(0,2), \sigma>0$, define

$$
H=H(\lambda, \sigma) \stackrel{\text { def }}{=} \frac{\lambda}{2-\lambda}(2 \sigma-m)-1 .
$$


Assume that $H>0, \vartheta H<2+\vartheta(m-1)$. Then we have, for all $B_{\rho} \subset \mathbf{R}^{N}$, $0<t<T, R(t) \leq \rho \leq 1$

$$
\int_{0}^{t} \int_{B_{\rho / 2}}\left|D u^{\sigma}\right|^{\lambda} d x d \tau \leq \gamma U(t)\left\{\Gamma^{\frac{2}{\mathcal{K}}(N-\vartheta) H}\langle u\rangle_{t}^{\frac{2 H}{\mathcal{K}}} t^{1-\frac{\vartheta}{2+\vartheta(m-1)} H}\right\}^{\frac{2-\lambda}{2}},
$$

where $U(t)=\sup _{0<\tau<t}\|u(\cdot, \tau)\|_{1, B_{\rho}}$. In $(2.6) \gamma=\gamma(N, m, \nu, p, \lambda, \sigma, \vartheta)$.

Proof. Let $R(t) \leq \rho \leq 1$, and choose in (1.5) the testing function $\eta=t^{\beta} \zeta^{2} u^{r}$, with $\zeta=\zeta(x)$ a standard cutoff function in $B_{\rho}, \zeta \equiv 1$ in $B_{\rho / 2},|D \zeta| \leq \gamma / \rho$. Here $\beta>0$, $r>0$ are to be chosen. The left-hand side of (1.5) is then bounded below by

$$
\gamma_{0} \iint|D u|^{2} u^{r+m-2} \tau^{\beta} \zeta^{2} d x d \tau-\gamma \iint \rho^{-2} u^{r+m} \tau^{\beta} d x d \tau-\gamma \iint \tau^{\beta-1} u^{1+r} d x d \tau .
$$

(All the double integrals in the present proof are calculated over $B_{\rho} \times(0, t)$.) We are assuming that $\beta, r$ are such that the integrals in (2.7) are finite (see (2.9) below). The right-hand side of (1.5) can be bounded above by means of Young's inequality, as in (2.3), to get, with the help of (2.7),

$$
\begin{aligned}
\iint \tau^{\beta} \zeta^{2} u^{r+m-2}|D u|^{2} d x d \tau & \leq \gamma \iint \tau^{\beta-1} u^{1+r}\left(1+\tau \rho^{-2} u^{m-1}+\tau u^{\Theta}\right) d x d \tau \\
& \leq \gamma \iint u^{1+r} \tau^{\beta-1} d x d \tau \stackrel{\text { def }}{=} J_{1} .
\end{aligned}
$$

In the last majorization we have made use of assumption (2.1). Owing to the sup estimate provided by Lemma 2.1 we may further bound $J_{1}$ above by

$$
J_{1} \leq \gamma U(t) \int_{0}^{t} \tau^{\beta-1}\|u(\cdot, \tau)\|_{\infty, B \rho}^{r} d \tau \leq \gamma U(t) \Gamma^{\frac{2}{\mathcal{K}}(N-\vartheta) r}\langle u\rangle_{t}^{\frac{2}{\mathcal{K}} r} t^{\beta-\frac{\vartheta}{2+\vartheta(m-1)}} r \stackrel{\text { def }}{=} J_{2},
$$

provided

$$
\beta>\vartheta r /(2+\vartheta(m-1)) .
$$

Next we estimate by Hölder's inequality and by (2.8)

$$
\begin{aligned}
& \iint\left|D u^{\sigma}\right|^{\lambda} \zeta^{\frac{1}{\lambda}} d x d \tau \\
& \quad \leq\left(\iint|D u|^{2} \tau^{\beta} u^{r+m-2} \zeta^{2} d x d \tau\right)^{\frac{\lambda}{2}}\left(\iint \tau^{-\beta \frac{\lambda}{2-\lambda}} u^{1+H-r \frac{\lambda}{2-\lambda}} d x d \tau\right)^{1-\frac{\lambda}{2}} \\
& \quad \leq J_{2}^{\frac{\lambda}{2}}\left(\iint \tau^{-\beta \frac{\lambda}{2-\lambda}} u^{1+H-r \frac{\lambda}{2-\lambda}} d x d \tau\right)^{1-\frac{\lambda}{2}} .
\end{aligned}
$$

Assume from now on

$$
\lambda r /(2-\lambda) \leq H
$$

Then, we get

$$
\begin{gathered}
\iint \tau^{-\beta \frac{\lambda}{2-\lambda}} u^{1+H-r \frac{\lambda}{2-\lambda}} d x d \tau \leq U(t) \int_{0}^{t} \tau^{-\beta \frac{\lambda}{2-\lambda}}\|u(\cdot, \tau)\|_{\infty, B \rho}^{H-r \frac{\lambda}{2-\lambda}} d \tau \\
\leq \gamma U(t) \Gamma^{\frac{2}{\mathcal{K}}(N-\vartheta)\left(H-r \frac{\lambda}{2-\lambda}\right)}\langle u\rangle_{t}^{\frac{2}{\mathcal{K}}\left(H-r \frac{\lambda}{2-\lambda}\right)} t^{\xi},
\end{gathered}
$$


provided

$$
\xi \stackrel{\text { def }}{=} 1-\frac{\vartheta}{2+\vartheta(m-1)} H-\frac{\lambda}{2-\lambda}\left(\beta-\frac{\vartheta}{2+\vartheta(m-1)} r\right)>0 .
$$

Note that by substituting (2.12) in (2.10), we get (2.6) after easy calculations. It is therefore only left to prove that $\beta, r$ can be chosen so as to fulfill (2.9), (2.11), (2.13). This is a trivial task if $\vartheta=0$. Assume then $\vartheta>0$, and fix arbitrarily

$$
0<\beta<(2-\lambda) / \lambda \text {. }
$$

We write (2.13) as

$$
r>\left[\frac{2-\lambda}{\lambda}\left(\frac{\vartheta}{2+\vartheta(m-1)} H-1\right)+\beta\right] \frac{2+\vartheta(m-1)}{\vartheta} \stackrel{\text { def }}{=} z_{0},
$$

and we combine (2.9), (2.11) in the form

$$
r<\min \left(\frac{2+\vartheta(m-1)}{\vartheta} \beta, \frac{2-\lambda}{\lambda} H\right) \stackrel{\text { def }}{=} z_{1} .
$$

It is easy to check that $z_{0}<z_{1}$, as a consequence of the assumption $\vartheta H<2+$ $\vartheta(m-1)$ and of (2.14). Finally we fix $r>0$ such that $r \in\left(z_{0}, z_{1}\right)$. This concludes the proof.

Remark 2.1. A version of Lemma 2.2 holds even if $H=0$. Indeed, an inspection of the proof shows that we only need replace $H$ with any $h>0, \vartheta h<(2+\vartheta(m-1))$ in (2.6), and in (2.11)-(2.16). Also, $U(t)$ is substituted with $U(t)^{1-h(1-\lambda / 2)}$ in (2.6), and with $U(t)^{1-h}$ in (2.12).

From now on we specialise the choice of the constant $\Gamma$ appearing in the definition of $R(t)$ to

$$
\Gamma=C[\mu]^{\frac{m-1}{2+\vartheta(m-1)}},
$$

where $C>0$ will be chosen below as a function of $N, p, m, \nu$, and $\vartheta$. (Obviously we may assume $[\mu]>0$ throughout.)

Remark 2.2. By definition it holds $\langle u\rangle_{t} \leq[u]_{t}$. Moreover, using Lemma 2.1 to estimate the integrals over $B_{\rho}$, with $0<\rho<R(\tau)$, appearing in the definition of $[u]_{t}$, we find also

$$
[u]_{t} \leq\langle u\rangle_{t}+\gamma(C)[\mu]^{\frac{N}{K}(m-1)}\langle u\rangle_{t}^{\frac{2}{K}}, \quad 0<t<T .
$$

Here $T$ is chosen as in Lemma (2.1), and we have used (2.17) too.

Let us conclude this section with the following lemma, giving a priori bounds of a (regular) solution to (1.1) in terms of the initial datum.

Lemma 2.3. Let $u \geq 0$ be a uniformly continuous and bounded solution to (1.1) in $S$. Then if $\vartheta(p \nu-m)<(2-\nu)$, there exists a $T_{0}>0, T_{0}=T_{0}([\mu], N, m, \nu, p, \vartheta)$, such that

$$
[u]_{t} \leq \gamma[\mu], \quad 0<t<T_{0},
$$

and (2.1), (2.2) hold for $0<t<T_{0}$. Here $\gamma=\gamma(N, m, \nu, p, \vartheta)$. 
Proof. Define

$$
t_{0}=\sup \left\{0<T<T^{*} \mid(2.1) \text { holds, where } \Gamma \text { is given by }(2.17)\right\},
$$

and assume $m>1$ for the sake of definiteness; the case $m=1$ can be handled similarly, recalling Remark 2.1. Choose $0<t<t_{0}$, and let $B_{\rho} \subset \mathbf{R}^{N}$ be any ball with radius $R(t) \leq \rho \leq 1$, centered at an arbitrarily fixed $x_{0} \in \mathbf{R}^{N}$. If $\zeta$ is a standard cutoff function in $B_{\rho}, \zeta \equiv 1$ in $B_{\rho / 2}$, we find after straightforward calculations

$$
\int_{B_{\rho / 2}} u(x, t) d x \leq \int_{B_{\rho}} d \mu+\frac{\gamma}{\rho} \int_{0}^{t} \int_{B_{\rho}}\left|D u^{m}\right| d x d \tau+\int_{0}^{t} \int_{B_{\rho}}\left|D u^{p}\right|^{\nu} d x d \tau
$$

On multiplying both sides by $\rho^{\vartheta}\left|B_{\rho}\right|^{-1}$ we find employing Lemma 2.2 ,

$$
\begin{aligned}
\rho^{\vartheta} f_{B_{\rho / 2}} u(x, t) d x \leq 2^{N}[\mu]+\gamma\langle u\rangle_{t} & \left\{C^{-\frac{2+\vartheta(m-1)}{\mathcal{K}}\left(\frac{\langle u\rangle_{t}}{[\mu]}\right)^{\frac{m-1}{\mathcal{K}}}}\right. \\
& \left.+\left(\Gamma^{\frac{2}{\mathcal{K}}(N-\vartheta) \Theta}\langle u\rangle_{t}^{\frac{2}{\mathcal{K}} \Theta} t^{1-\frac{\vartheta}{2+\vartheta(m-1)} \Theta}\right)^{\frac{2-\nu}{2}}\right\},
\end{aligned}
$$

for all $0<t<t_{0}, R(t) \leq \rho \leq 1$. Taking into account that $x_{0} \in \mathbf{R}^{N}$ has been chosen arbitrarily, it is immediately seen, by a standard covering argument, that we have in fact proven

$$
\langle u\rangle_{t} \leq \gamma_{1}(N)[\mu]+\gamma_{2}\langle u\rangle_{t}\left\{\mathcal{L}\left(C,\langle u\rangle_{t}\right)+\mathcal{M}\left(C, t,\langle u\rangle_{t}\right)\right\}
$$

where the meaning of $\mathcal{L}$ and $\mathcal{M}$ is obvious. Let us remark that $\gamma_{2}$ in (2.18) depends on the same quantities determining the constants $\gamma$ in Lemmata 2.1, and 2.2, but it does not depend on $C$. Next we define

$$
t_{1}=\sup \left\{0<t<T^{*} \mid\langle u\rangle_{t} \leq 4 \gamma_{1}[\mu]\right\}, \quad t_{2}=\sup \left\{0<t<T^{*} \mid \mathcal{M}\left(C, t,\langle u\rangle_{t}\right)<\delta\right\},
$$

where the (small) constant $\delta>0$ is to be chosen. Note that $t_{1}, t_{2}$ are well defined because the stipulated assumptions make sure that $\langle u\rangle_{t}$ is continuous in $\left[0, T^{*}\right]$, and that the exponent of $t$ in $\mathcal{M}$ is positive. Let $t_{3}=\min \left(t_{0}, t_{1}, t_{2}\right)$. Then for $0<t<t_{3}$ we have

$$
\gamma_{2} \mathcal{L}\left(C,\langle u\rangle_{t}\right) \leq \gamma_{2} C^{-\frac{2+\vartheta(m-1)}{\mathcal{K}}}\left(4 \gamma_{1}\right)^{\frac{m-1}{\mathcal{K}}} \leq 1 / 4
$$

provided $C$ is suitably chosen. Then, if we also choose $\delta<\left(4 \gamma_{2}\right)^{-1}$, it follows from

$$
\langle u\rangle_{t} \leq 2 \gamma_{1}[\mu], \quad 0<t<t_{3}
$$

As noted in Remark 2.2, (2.20), together with Lemma 2.1, provides us with the sought after estimate for $[u]_{t}$. Therefore all the claims made in the statement will follow, using the sup estimates proven in this section, provided we show that we can indeed find a quantitative estimate below $t_{3} \geq T_{0}$, with $T_{0}$ as above. We may assume $t_{3}<T^{*}$, since the estimate is otherwise trivial. First, we note that $(2.20)$ implies that $t_{3}<t_{1}$. Next, let us rule out the case $t_{3}=t_{0}$. To this end, we show that, for a suitable choice of $C$ and $\delta$, the left-hand side of (2.1) is bounded away 
from 1 up to $t=t_{3}$. As a matter of fact, Lemma 2.1, together with (2.20), implies for $t \leq t_{3}, x \in \mathbf{R}^{N}$,

$$
\begin{aligned}
& \Gamma^{-2} t^{\frac{\vartheta}{2+\vartheta(m-1)}}(m-1) u^{m-1}(x, t)+t u^{\Theta}(x, t) \\
& \quad \leq \gamma C^{-\frac{2}{\mathcal{K}}(2+\vartheta(m-1))}+\gamma \mathcal{M}\left(C, t,\langle u\rangle_{t}\right)^{\frac{2}{2-\nu}} \\
& \quad \leq \gamma\left(C^{-\frac{2}{\mathcal{K}}(2+\vartheta(m-1))}+\delta^{\frac{2}{2-\nu}}\right) \leq 1 / 2,
\end{aligned}
$$

if $C$ is chosen large enough, and then $\delta$ in the definition of $t_{2}$ is chosen small enough. Let us remark that this can be done safely, as the constant $\gamma$ in (2.21) is known a priori, and, especially, it does not depend on $C$ or $\delta$. Finally, we are left with the task of estimating below $t_{3}=t_{2}$. But this can be accomplished at once, by replacing $\langle u\rangle_{t}$ with $2 \gamma_{1}[\mu]$ in the definition of $t_{2}$, owing to $(2.20)$.

\section{Proof of Theorem 1.1}

We construct a sequence of approximating problems for $n=1,2, \ldots$,

$$
\begin{gathered}
u_{n t}-\Delta u_{n}^{m}=\min \left(\left|D u_{n}^{p}\right|^{\nu}, n\right), \quad \text { in } \mathbf{R}^{N} \times(0, \infty) ; \\
u_{n}(x, 0)=u_{0 n}(x), \quad x \in \mathbf{R}^{N},
\end{gathered}
$$

with $u_{0 n} \geq 1 / n, u_{0 n} \in C^{\infty}\left(\mathbf{R}^{N}\right) \cap L^{\infty}\left(\mathbf{R}^{N}\right), u_{0 n} \rightarrow \mu$ in the sense of measures as $n \rightarrow \infty$. We can assume without loss of generality that $\left[u_{0 n}\right] \leq \gamma(N)[\mu]$. Existence of a smooth solution $u_{n}$ to (3.1) follows from standard parabolic theory. Let us remark that the estimates in section 2 can be applied to $u_{n}$, because it is a smooth subsolution to (1.1a); even Lemma 2.3 holds true for each $u_{n}$, its proof being based upon the a priori estimates of Lemmata 2.1 and 2.2. Then, by the mentioned a priori estimates and by standard calculations, we find

$$
\left\|u_{n}\right\|_{\infty, K} \leq \gamma(K,[\mu]) ; \quad\left\|D u_{n}^{\frac{m+q}{2}}\right\|_{2, K} \leq \gamma(K,[\mu], q), \quad \text { for all } q \geq 0,
$$

for any compact set $K \subset S_{T_{0}}=\mathbf{R}^{N} \times\left(0, T_{0}\right)$ where $T_{0}$ is defined as in Lemma 2.3. Note that $T_{0}$, and the bounds in (3.2), do not depend on $n$. As a consequence of the quoted results of [9], one gets uniform Hölder estimates for the sequence $\left\{u_{n}\right\}$, in each compact set contained in $S_{T_{0}}$. Then we may assume

$$
u_{n} \rightarrow u \text { uniformly on compact subsets of } S_{T_{0}} .
$$

In order to prove (1.5), we of course need take the limit $n \rightarrow \infty$ in the corresponding weak formulation of (3.1). The uniform estimates (3.2), and (3.3), allow us to take immediately the limit in the left-hand side of (1.5). The right-hand side, being nonlinear in $D u$, requires further discussion.

Let us consider first the case $m>1$, Multiply the equation in (3.1) against $u_{n}^{s-1}\left(u_{n}^{s}-u_{k}^{s}\right) \zeta^{2}$, where $n, k \geq 1$ are arbitrary, $s>1$ is to be chosen, and $\zeta$ is a standard compactly supported cutoff function in $S_{T_{0}}$. We get by integration by parts

$$
\begin{aligned}
& \iint\left\{\frac{1}{s}\left(u_{n}^{s}\right)_{t}\left(u_{n}^{s}-u_{k}^{s}\right) \zeta^{2}+D u_{n}^{m} \cdot D\left[u_{n}^{s-1}\left(u_{n}^{s}-u_{k}^{s}\right) \zeta^{2}\right]\right\} d x d t \\
& =\iint \min \left(\left|D u_{n}^{p}\right|^{\nu}, n\right) u_{n}^{s-1}\left(u_{n}^{s}-u_{k}^{s}\right) \zeta^{2} d x d t .
\end{aligned}
$$


We add (3.4) to the similar equality obtained by interchanging $u_{n}$ with $u_{k}$. On integrating by parts once more, we find after obvious simplifications

$$
\begin{aligned}
& \left|\iint\left\{D u_{n}^{m} \cdot D\left[u_{n}^{s-1}\left(u_{n}^{s}-u_{k}^{s}\right) \zeta^{2}\right]-D u_{k}^{m} \cdot D\left[u_{k}^{s-1}\left(u_{n}^{s}-u_{k}^{s}\right) \zeta^{2}\right]\right\} d x d t\right| \\
& \quad \leq \gamma \iint\left\{\left|\zeta_{t}\right|+\left|D u_{n}^{p}\right|^{\nu} u_{n}^{s-1}+\left|D u_{k}^{p}\right|^{\nu} u_{k}^{s-1}\right\}\left|u_{n}^{s}-u_{k}^{s}\right| \zeta d x d t \rightarrow 0, \quad n, k \rightarrow \infty .
\end{aligned}
$$

The limit relation in (3.5) follows from the sup and gradient estimates valid for the whole sequence $\left\{u_{n}\right\}$, and of course from (3.3). Elementary manipulations of the integrand in (3.5) show that, for the choice $s=(m+1) / 2$, we have

$$
\iint\left|D u_{n}^{m}-D u_{k}^{m}\right|^{2} \zeta^{2} d x d t \rightarrow 0, \quad n, k \rightarrow \infty .
$$

Therefore, by extracting a subsequence if necessary, we have

$$
D u_{n}^{m} \rightarrow D u^{m}, \quad \text { a.e. in } S_{T_{0}} \text {, and in } L_{\mathrm{loc}}^{2}\left(S_{T_{0}}\right) .
$$

If $m=1$, (3.6) may be proven exactly as above, with the choice $s=1$.

By means of standard arguments in real analysis, (1.5) will be a consequence of the uniform integrability (3.2) for the sequence $\left\{\left|D u_{n}^{p}\right|^{\nu}\right\}$, provided we show that $\left|D u_{n}^{p}\right|^{\nu} \rightarrow\left|D u^{p}\right|^{\nu}$ a.e. in $S_{T_{0}}$. Clearly, this is immediately implied by (3.3), (3.6) if $p \geq m$. If $m / \nu \leq p<m$, it is only left to check

$$
D u_{n}^{p} \rightarrow 0 \quad \text { a.e. in }\{u=0\} .
$$

Let us note that $\left\{\left|D u_{n}^{\frac{m}{2}}\right|^{\nu}\right\}$ is uniformly bounded in $L_{\text {loc }}^{\frac{2}{\nu}}\left(S_{T_{0}}\right)$, by virtue of (3.2). Thus, without loss of generality, we may assume

$$
\left|D u_{n}^{\frac{m}{2}}\right|^{\nu} \rightarrow \Xi, \quad \text { weakly in } L_{\text {loc }}^{\frac{2}{\nu}}\left(S_{T_{0}}\right) .
$$

But then

$$
\left|D u_{n}^{p}\right|^{\nu}=(2 p / m)^{\nu}\left|D u_{n}^{\frac{m}{2}}\right|^{\nu} u_{n}^{\nu\left(p-\frac{m}{2}\right)} \rightarrow(2 p / m)^{\nu} \Xi u^{\nu\left(p-\frac{m}{2}\right)}, \quad \text { weakly in } L_{\text {loc }}^{\frac{2}{\nu}}\left(S_{T_{0}}\right) .
$$

Taking into account that the sequence $\left\{\left|D u_{n}^{p}\right|^{\nu}\right\}$ is nonnegative, we infer that $\left|D u_{n}^{p}\right|^{\nu} \rightarrow 0$ in $L_{\text {loc }}^{1}\left(S_{T_{0}} \cap\{u=0\}\right)$, and (3.7) follows, by extracting a suitable subsequence if necessary. The convergence $u(\cdot, t) \rightarrow \mu, t \rightarrow 0$, in the sense of measures, can be proven easily, exploiting the uniform integrability of $\left|D u_{n}^{p}\right|^{\nu}$ and $\left|D u_{n}^{m}\right|$ up to $t=0$, provided by Lemma 2.2 (with $\rho=1$ ).

\section{Proof of Theorem 1.2}

Of course we may assume $\vartheta>0, p \nu>m$, and $x_{0}=0$; from now on, all the balls are understood to be centered at 0 . Let $w$ be the first eigenfunction of

$$
-\Delta w=\lambda w, \quad \text { in } B_{\rho}, \quad w=0, \quad \text { on } \partial B_{\rho},
$$

where we may assume $\|w\|_{1, B_{\rho}}=1, \gamma \rho^{-N} \geq w \geq 0$, and $w \geq \gamma_{0} \rho^{-N}$ in $B_{\rho / 2}$. Let $\lambda_{1}=\gamma(N) \rho^{-2}$ be the corresponding eigenvalue. From Definition 1.1 we get for $0<t^{\prime}<t<T, \rho>0$,

$$
\int_{B_{\rho}(t)} u w d x-\int_{B_{\rho}\left(t^{\prime}\right)} u w d x \geq-\frac{\gamma}{\rho^{2}} \int_{t^{\prime}}^{t} \int_{B_{\rho}} u^{m} w d x d \tau+\int_{t^{\prime}}^{t} \int_{B_{\rho}}\left|D u^{p}\right|^{\nu} w d x d \tau
$$


where we let $B_{\rho}(t)=B_{\rho} \times\{t\}$. Next we note that, due to our assumption $[u]_{t}<\infty$, we have

$$
\int_{B_{\rho}(t)} u d x \leq \gamma \rho^{N-\vartheta}, \quad 0<t<t_{*}, 0<\rho<1,
$$

for a suitable $t_{*}>0$. Define

$$
A(t)=\left\{x \in B_{\rho} \mid u(x, t)>\omega \rho^{-\vartheta}\right\}, \quad E(t)=B_{\rho} \backslash A(t),
$$

$\omega>0$ to be chosen. Hence for $0<t<t_{*}$, (4.2) together with Chebychev's inequality yields

$$
|A(t)| \leq \gamma \omega^{-1}\left|B_{\rho}\right|, \quad 0<t<t_{*}, \quad 0<\rho<1 .
$$

As a consequence

$$
\left|E(t) \cap B_{\rho / 2}\right| \geq \frac{1}{2}\left|B_{\rho / 2}\right|, \quad 0<t<t_{*}, \quad 0<\rho<1,
$$

if $\omega$ is chosen large enough.

Let us state the following result, which can be proven as Lemma 5.1 in [15], p. 89: Let $v \in W_{q}^{1}\left(B_{\rho}\right), v \geq 0, q \geq 1$. Then for all measurable sets $\mathcal{E}_{0} \subset B_{\rho / 2}, v \equiv 0$ in $\mathcal{E}_{0}$, we have

$$
\int_{B_{\rho}} v^{q}(x) G(|x|) d x \leq \gamma(N, q)\left(\rho^{N+1}\left|\mathcal{E}_{0}\right|^{-1} G(0) G(\rho / 2)^{-1}\right)^{q} \int_{B_{\rho}}|D v(x)|^{q} G(|x|) d x,
$$

where $G:[0, \rho) \rightarrow(0, \infty)$ is nonincreasing.

On letting $v=\left(u-\omega \rho^{-\vartheta}\right)_{+}^{p}, q=\nu, \mathcal{E}_{0}=E(t) \cap B_{\rho / 2}, G(|x|)=w(x)$, we find

$$
\gamma \int_{t^{\prime}}^{t} \int_{B_{\rho}}\left|D u^{p}\right|^{\nu} w d x d \tau \geq \rho^{-\nu} \int_{t^{\prime}}^{t} \int_{B_{\rho}}\left(u-\omega \rho^{-\vartheta}\right)_{+}^{p \nu} w d x d \tau
$$

for $0<t^{\prime}<t<t_{*}, 0<\rho<1$. Moreover, we note that (1.4) implies

$$
u(x, \tau) \geq 2 \omega \rho^{-\vartheta}, \quad t / 2<\tau<t,|x| \leq \gamma_{0} \rho, \quad \text { for } \rho=\rho(t)=P_{o} t^{\frac{1}{2+\vartheta(m-1)}},
$$

provided $P_{0}>0$ is fixed large enough (in (4.4) $\gamma_{0}$ depends on $P_{0}$ ). From now on, we choose $\rho$ as in (4.4). We also redefine $t^{*}$ to ensure $\rho<1$ for $t<t^{*}$. Thus straightforward calculations lead us to

$$
\gamma \int_{B_{\rho}(\tau)}\left(u-\omega \rho^{-\vartheta}\right)_{+}^{p \nu} w d x \geq \int_{B_{\rho}(\tau)} u^{p \nu} w d x, \quad t / 2<\tau<t<t^{*}
$$

Collecting (4.1), (4.3), (4.5), we find, with the help of Young's inequality,

$$
\int_{B_{\rho}(z)} u w d x-\int_{B_{\rho}(t / 2)} u w d x \geq-\gamma \rho^{-\nu \frac{2 p-m}{\nu p-m}}(z-t / 2)+\gamma_{0} \rho^{-\nu} \int_{t / 2}^{z} \int_{B_{\rho}} u^{p \nu} w d x d \tau,
$$

for $t / 2<z<t$. Then, $\int_{B_{\rho}} u w$ majorizes the solution $y$ to

$$
y^{\prime}=\gamma_{0} \rho^{-\nu} y^{p \nu}-\gamma \rho^{-\nu \frac{2 p-m}{\nu p-m}}, \quad \text { in }(t / 2, t), \quad y(t / 2)=\int_{B_{\rho}(t / 2)} u w d x .
$$


Hence the solution $y$ to the nonlinear differential equation above is bounded over $(t / 2, t)$; it follows (see [2], Lemma 4.1) that

$$
\int_{B_{\rho}(t / 2)} u w d x \leq \gamma \max \left(\rho^{\frac{\nu}{p \nu-1}} t^{-\frac{1}{p \nu-1}}, \rho^{-\frac{2-\nu}{p \nu-m}}\right) .
$$

On the other hand, by (4.4) and by $w \geq \gamma_{0} \rho^{-N}$ in $B_{\rho / 2}$, we have

$$
\int_{B_{\rho}(t / 2)} u w d x \geq \gamma_{0} \rho^{-\vartheta}
$$

for $t$ small and $\rho=\rho(t)$ defined above. On letting $t \rightarrow 0$ it is easily seen that inequalities (4.6) imply $\nu<2$ and $\vartheta \leq(2-\nu) /(p \nu-m)$.

Remark 4.1. A quick glance at the proof shows that one can replace the assumption $[u]_{t}<\infty$ with its localized version (4.2).

\section{ACKNOWLEDGMENT}

The author is indebted to the referee for several useful suggestions.

\section{REFERENCES}

1. H. Amann, Nonhomogeneous linear and quasilinear elliptic and parabolic boundary value problems, Function spaces, differential operators and nonlinear analysis, Texte zur Math., 133, eds. H. Schmeisser and H. Triebel, Teubner, Stuttgart-Leipzig, 1993, 9-126. MR 94m:35153

2. D. Andreucci, New results on the Cauchy problem for parabolic systems and equations with strongly nonlinear sources, Manuscripta Math. 77 (1992), 127-159. MR 93k:35129

3. D. Andreucci, E. DiBenedetto, A new approach to initial traces in nonlinear filtration, Ann. Inst. H. Poincaré Anal. Non Linéaire 7 (1990), 305-334. MR 92g:35089

4. D. Andreucci, E. DiBenedetto, On the Cauchy problem and initial traces for a class of evolution equations with strongly nonlinear sources, Ann. Scuola Norm. Sup. Pisa 18 (1991), 363-441. MR 92m:35146

5. D. G. Aronson, L. A. Caffarelli, The initial trace of a solution of the porous medium equation, Trans. AMS 280 (1983), 351-366. MR 85c:25042

6. P. Baras, M. Pierre, Critère d'existence de solutions positives pour des équations semilinéaires non monotones, Ann. Inst. H. Poincaré Anal. Non linéaire, 2 (1985), 185-212. MR 87j:45032

7. Ph. Bènilan, M. G. Crandall, M. Pierre, Solutions of the porous medium equation in $\mathbf{R}^{N}$ under optimal conditions on initial values, Indiana Univ. Math. J., 33 (1984), 51-87. MR 86b:35084

8. L. Boccardo, T. Gallouët, Nonlinear elliptic and parabolic equations involving measure data, J. Funct. Anal. 87 (1989), 149-169. MR 92d:35286

9. E. DiBenedetto, A. Friedman, Hölder estimates for non-linear degenerate parabolic systems, J. Reine Angew. Math., 357 (1985), 1-22. MR 87f:35134a

10. Y. Giga, T. Miyakawa, Navier-Stokes flow in $\mathbf{R}^{3}$ with measures as initial vorticity and Morrey spaces, Comm. PDE 14 (1989), 577-618. MR 90e:35130

11. A. L. Gladkov, The Cauchy problem in classes of increasing functions for the equation of filtration with convection, Mat. Sbornik 186 (1995), 35-56 (transl. Sbornik Math. 186 (1995), 803-825). MR 96f:35093

12. H. Hoshino, Y. Yamada, Solvability and smoothing effect for semilinear parabolic equations, Funkcial. Ekvac., 34 (1991), 475-494. MR 93c:34131

13. K. Kobayasi, Semilinear parabolic equations with nonmonotone nonlinearity, Memoirs Sagami Inst. of Technology, 23 (1989), 83-99.

14. H. Kozono, M. Yamazaki, Semilinear heat equations and the Navier-Stokes equation with distributions in new function spaces as initial data, Comm. PDE 19 (1994), 959-1014. MR 95d:35077

15. O. A. Ladyzenskaja, V. A. Solonnikov, N. N. Ural'tzeva, Linear and quasilinear equations of parabolic type, Transl. Math. Monos., 23, AMS, Providence, R.I., 1967. MR 39:3159b 
16. Y. Niwa, Semilinear heat equations with measures as initial data, Thesis, University of Tokyo, 1986.

17. M. E. Taylor, Analysis on Morrey spaces and applications to Navier-Stokes and other evolution equations, Comm. PDE 17 (1992), 1407-1456. MR 94b:35218

Università "La Sapienza", Dipartimento di Metodi e Modelli Matematici, via A. Scarpa 16, 00161 Roma, Italy

E-mail address: andreucc@dmmm.uniroma1.it 